\title{
Approach towards Optimum Data Transfer with Various Automatic Variable Key (AVK) Techniques to Achieve Perfect Security with Analysis and Comparison
}

\author{
Rajat S Goswami \\ Department of CSE \\ NIT Arunachal \\ Pradesh, India
}

\author{
Swarnendu K \\ Chakraborty \\ Department of CSE \\ NIT Arunachal \\ Pradesh, India
}

\author{
Abhinandan \\ Bhunia \\ Microsoft Corporations, \\ USA
}

\author{
Chandan T Bhunia \\ Director \\ NIT Arunachal \\ Pradesh, India
}

\begin{abstract}
Session to session key variation is the only approach for achieving perfect security as per Shannon [1-2]. This paper deals with time variant key technique Automatic Variable Key (AVK) introduced by Bhunia[3-5] and we tried to analyze the performance of different types of new AVK techniques.
\end{abstract}

\section{Keywords}

AVK, CSAVK, ASAVK, DSAVK, KVRN Protocol, Randomness, Perfect Security.

\section{INTRODUCTION}

In all cryptosystem the Key challenge of the designer is to make key unbreakable. Shannon proposed that key would be impossible to break if the key is made time variant. The time variant key can be implemented by changing key from session to session. AVK was one of the noble approaches for achieving perfect security invented by Bhunia [3-5]. The superiority of time variant key in achieving perfect security is studied elsewhere [6-14].In AVK technique which illustrated in Table-1 the key is made variable by an agreement that creates new key for each data. This is reviewed as below:

Say, $\mathrm{K}_{0}=$ initial key that may be exchanged by any conventional secret mode between a sender and a receiver.

Subsequent keys for different data (Di-1) to be exchanged are generated are:

$\mathrm{K}_{\mathrm{i}}=\mathrm{K}_{\mathrm{i}-1}$ XOR $\mathrm{D}_{\mathrm{i}-1}$ for $\mathrm{i} \geq 0$.

The key is made variable with exchanged data between a sender and a receiver. A new key is generated every time a data is exchanged. The new key so generated is used subsequently for further exchange of data.

The illustrated technique of AVK has been extensively applied in both private and public key cryptography. The application is found to reduce brute force attack, frequency attack and differential frequency attack [7-14].

So far applied AVK is based on the generating function XOR as in equation (1).

In CSAVK [14] technique illustrated in below the key is made variable by one agreement that also creates new key for each data.

Say, $\mathrm{K}_{0}=$ initial key that may be exchanged by any conventional secret mode between a sender and a receiver.

Subsequent keys for different data $\left(D_{i-1}\right)$ to be exchanged are generated are:
$\mathrm{K}_{\mathrm{i}}=\mathrm{K}_{\mathrm{i}-1}^{\prime}$ XOR $\mathrm{D}_{\mathrm{i}-1}^{\prime}$ for $\mathrm{i} \geq 0$.

Table 1: Elucidation of application of simple AVK in cryptology

\begin{tabular}{|c|c|c|c|c|c|}
\hline $\begin{array}{l}\text { Sessio } \\
\text { n } \\
\text { slots }\end{array}$ & \begin{tabular}{|c|} 
Sender \\
sends \\
his /her \\
private \\
key to \\
receiver
\end{tabular} & $\begin{array}{c}\text { Receiver } \\
\text { recovers } \\
\text { private key } \\
\text { from } \\
\text { sender }\end{array}$ & $\begin{array}{c}\text { Receive } \\
\text { r sends } \\
\text { his / her } \\
\text { private key } \\
\text { to sender }\end{array}$ & $\begin{array}{l}\text { Sender } \\
\text { receives } \\
\text { private } \\
\text { key from } \\
\text { receiver }\end{array}$ & Remarks \\
\hline 1 & $\begin{array}{l}\text { Secret key } \\
\text { Say 5(101) }\end{array}$ & 101 & $\begin{array}{l}\text { A secret key } \\
\text { Say } 7(111)\end{array}$ & 111 & $\begin{array}{c}\text { For next } \\
\text { slot sender } \\
\text { will use } 111 \\
\text { as key } \\
\text { and receiver } \\
101 \text { as key } \\
\text { for } \\
\text { transmittin } \\
\text { g data }\end{array}$ \\
\hline 2 & $\begin{array}{c}\text { Sender } \\
\text { sends } \\
\text { first } \\
(\text { random 3) } \\
\text { data } \\
011 \oplus 111 \\
=100\end{array}$ & $\begin{array}{c}\text { Receiver } \\
\text { gets original } \\
\text { data } \\
011 \oplus 111 \\
\oplus 111=011\end{array}$ & $\begin{array}{c}\text { Receive } \\
\mathrm{r} \text { sends } \\
\text { first } \\
\text { (random } \\
\text { data 9) as } \\
1001 \\
\oplus 0101=110 \\
0\end{array}$ & 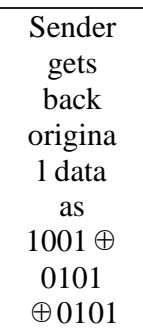 & 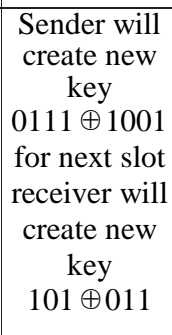 \\
\hline 3 & $\begin{array}{c}\text { Sender } \\
\text { sends } \\
\text { new } \\
\text { data } \\
4(100) \\
\text { as } 0100 \\
\oplus 0111 \\
\oplus 1001\end{array}$ & $\begin{array}{c}\text { Receive } \\
r \\
\text { recovers } \\
\text { original } \\
\text { data as } \\
0100 \oplus 0111 \\
\oplus 1001 \oplus 011 \\
1 \\
\oplus 1001= \\
0100\end{array}$ & $\begin{array}{c}\text { Receive } \\
\text { r sends } \\
\text { next data } 8 \\
(1000) 1000 \\
\oplus \\
0101 \oplus 0011\end{array}$ & $\begin{array}{l}\text { Sender } \\
\text { receive } \\
\mathrm{s} \\
\text { original } \\
\quad \text { data } \\
1000 \\
\oplus 0101 \\
\oplus 0011 \\
\oplus 0101 \\
\oplus 0011 \\
=1000\end{array}$ & $\begin{array}{c}\text { Sender } \\
\text { computes } \\
\text { new } \\
\text { key } \\
011 \oplus 100 \\
\text { receiver } \\
\text { computes } \\
\text { key } \\
1001 \oplus 1000 \\
\text { for } \\
\text { transmittin } \\
\text { g next data }\end{array}$ \\
\hline
\end{tabular}

In ASAVK [14] technique illustrated in below the key is made variable by one another agreement that also creates new key for each data.

- Initial key $\left(\mathrm{K}_{0}\right)$ is exchanged between the sender and the receiver.

- Subsequent key, $\mathrm{K}_{\mathrm{i}}$ (at $\mathrm{i}^{\text {th }}$ stage) is generated by both sender \& receiver as : 
Step1:

$$
\mathrm{K}_{\mathrm{i}}=\mathrm{K}_{\mathrm{i}-1}^{\prime} \text { XOR } \mathrm{D}_{\mathrm{i}-1} \text { for } \mathrm{i} \geq 0 \text {. }
$$

Step2:

$\mathrm{K}_{\mathrm{i}+1}=\mathrm{K}_{\mathrm{i}-1}$ XOR D $_{\mathrm{i}-1}^{\prime}$ for $\mathrm{i} \geq 0$

Where $K_{i-1}^{\prime}=$ Block wise shift (Circular) $K_{i-1} /$ the number of shift will be total length of $\mathrm{K}_{\mathrm{i}-1}$ divided by 2 .

$D_{i-1}^{\prime}=$ Block wise shift (Circular) $D_{i-1} /$ the number of shift will be total length of $D_{i-1}$ divided by 2 .

In DSAVK [12] technique illustrated in below the key is made variable by another agreement that also creates new key for each data.

- Initial key $\left(\mathrm{K}_{0}\right)$ is exchanged between the sender and the receiver.

- $\quad$ Subsequent key, $\mathrm{K}_{\mathrm{i}}$ (at $\mathrm{i}^{\text {th }}$ stage) is generated by both sender $\&$ receiver as :

$\mathrm{K}_{\mathrm{i}}=\mathrm{K}_{\mathrm{i}-1}^{\prime}$ XOR $\mathrm{D}_{\mathrm{i}-1}$ for $\mathrm{i} \geq 0$.

Where $K_{i-1}^{\prime}=$ Bit wise right shift (Circular) $K_{i-1} /$ the number of shift will be the corresponding decimal value of $K_{i-1}$ XOR $D_{i-1}$.

In KVRN [14] technique illustrated in below the key is made variable by another agreement that also creates new key for each data.

- Initial key $\left(\mathrm{K}_{0}\right)$ and one numeric value $(\mathrm{m})$ is exchanged between the sender and the receiver.

- Subsequent key, $\mathrm{K}_{\mathrm{i}}$ (at $\mathrm{i}^{\text {th }}$ stage) is generated by both sender $\&$ receiver as :

$\mathrm{K}_{\mathrm{i}}=\mathrm{K}_{\mathrm{i}-1}+\mathrm{X}$ for $\mathrm{i} \geq 0$ and $\mathrm{X}=1$ to $\mathrm{m}$

Where $\mathrm{K}_{\mathrm{i}-1}=$ Previous key

When $\mathrm{X}=\mathrm{m}$, another key $\left(\mathrm{K}_{\mathrm{m}}\right)$ and another numeric value (n) is exchanged between the sender and the receiver.

Subsequent key will be generated same way as eqn. 6 .

In Protocol-I [13] technique illustrated in below the key is made variable by another agreement that also creates new key for each data.

- Initial key $\left(\mathrm{K}_{0}\right)$ and one noise burst $(\mathrm{m})$ is exchanged between the sender and the receiver by RSA.

- Subsequent key, $\mathrm{K}_{\mathrm{i}}$ (at $\mathrm{i}^{\text {th }}$ stage) is generated by both sender \& receiver as :

$\mathrm{K}=\mathrm{K}_{\mathrm{i}-1}$ XOR $\mathrm{D}_{\mathrm{i}-1}$ (AVK technique) for $\mathrm{i} \geq 0$

When $\mathrm{X}=\mathrm{m}$, another key $(\mathrm{Km})$ and another noise burst ( $\mathrm{n}$ ) is exchanged between the sender and the receiver.

Subsequent key will be generated same way as eqn.

$7 \&$ process will repeat.

In Protocol-II [13] technique illustrated in below the key is made variable by another agreement that also creates new key for each data.

- Initial key $\left(\mathrm{K}_{0}\right)$ and one noise burst $(\mathrm{m})$ is exchanged between the sender and the receiver by RSA.

- Subsequent key, $\mathrm{K}_{\mathrm{i}}$ (at $\mathrm{i}^{\text {th }}$ stage) is generated by both sender \& receiver as :

$\mathrm{K}_{\mathrm{i}+1}=\mathrm{K}_{\mathrm{i}}^{\prime} \mathrm{XOR}_{\mathrm{D}}^{\prime}{ }_{\mathrm{i}}(\mathrm{CSAVK}$ technique) for $\mathrm{i} \geq 0$... (8) When $\mathrm{X}=\mathrm{m}$, another key $\left(\mathrm{K}_{\mathrm{m}}\right)$ and another noise burst (n) is exchanged between the sender and the receiver. Subsequent key will be generated same way as eqn. $8 \&$ process will repeat.
In Protocol-III [13] technique illustrated in below the key is made variable by another agreement that also creates new key for each data.

- Initial key $\left(\mathrm{K}_{0}\right)$ and one noise burst $(\mathrm{m})$ is exchanged between the sender and the receiver by RSA.

- Subsequent key, $\mathrm{K}_{\mathrm{i}}$ (at $\mathrm{i}^{\text {th }}$ stage) is generated by both sender \& receiver as :

$\mathrm{K}_{\mathrm{i}}=\mathrm{K}_{\mathrm{i}-1}^{\prime} \quad$ XOR $\mathrm{D}_{\mathrm{i}-1}$ (DSAVK technique) for $\mathrm{i} \geq 0$...... (9)

When $\mathrm{X}=\mathrm{m}$, another key $(\mathrm{Km})$ and another noise burst (n) is exchanged between the sender and the receiver. Subsequent key will be generated same way as eqn. $8 \&$ process will repeat.

The key is made variable with exchanged data between a sender and a receiver every time a data is exchanged. The new key so generated is used subsequently for further exchange of data.

\section{NEW IDEA}

So far in AVK techniques, the main lacuna is to keep the initial key highly secret. In AVK, initial Key is distributed between sender by RSA or by key distribution centre.

We propose a new technique to exchange the initial key of AVK as follows:

Instead of sending one key, we proposed to send three keys. Say "101010101","11001001" and "00100110" are the initial keys exchanged between sender \& receiver by RSA. Sender \& Receiver will perform bit-wise majority logic operation among three keys to agree upon to the first key. In the example, therefore the first key is generated as;

10101010

11001001

00100110

10101010, after majority logic original initial key under different AVK techniques will be "10101010". The superiority of the proposed technique used in AVK is justified in terms of the probability of Key-breaking. In the probability of Key-breaking is $\mathrm{P}$, as in normal AVK, the same for the proposed technique is $\mathrm{P}^{3}(<\mathrm{P})$.

\section{Illustration of AVK, CSAVK, ASAVK, DSAVK, PROTOCOL-I, PROTOCOL-II and PROTOCOL-III}

\subsection{Illustration of AVK}

Let we assume that sender sends original data $\left(\mathrm{D}_{0}\right) 00000100$ in encrypted form using an initial key $\left(\mathrm{K}_{0}\right)=10101010$. Then in order to maintain the linearity, the encrypted form is 00000100 XOR $10101010=10101110$.

At receiver end receiver will perform 10101110 XOR 10101010 and gets 00000100 .

\subsection{Illustration of CSAVK}

Let sender sends initial data $\mathrm{D}_{0}(01010101)$ in encrypted form using key $\mathrm{K}_{0}(10101010)$. As per technique of CSAVK of eqn. (2) next key will be generated as $K_{0}=10101010$. The process will then be continued.

But in the next data transmission key will be changed by left shifting the previous data $\left(D_{0}\right)$ up to the total number of 1 's present in that data $\left(\mathrm{SD}_{0}\right)$ XOR with right shifting the 
previous key $\left(\mathrm{K}_{0}\right)$ up to the total number of 1's present in that key $\left(\mathrm{SK}_{0}\right)$.

So the new key will be $\mathrm{K}_{1}=\mathrm{SD}_{0}+\mathrm{SK}_{0}=00001000 \mathrm{XOR}$ $10101010=10100010$.

\subsection{Illustration of ASAVK}

Let sender sends initial data $\mathrm{D}_{0}(00000000)$ in encrypted form using key K (11001010). By the technique of ASAVK as in eqn.(3-4), the next key will be generated by block shift operation and will be $\mathrm{K}_{0}=10101100$.

But in the next data transmission key will be, block shifting of the previous data $\left(\mathrm{D}_{0}\right)$ named as $\left(\mathrm{SD}_{0}\right)$ XOR with the previous key $\left(\mathrm{K}_{0}\right)$.

So the new key will be $\mathrm{K}_{1}=\mathrm{SD}_{0}+\mathrm{K}_{0}=00000000 \mathrm{XOR}$ $10101100=10101100$.

\subsection{Illustration OF DSAVK}

Let sender sends initial data $\mathrm{D}_{0}(00000000)$ in encrypted form using key K (00000110). By the technique of DSAVK as in eqn.5, the next key will be generated by right shift operation and will be $\mathrm{K}_{0}=00011000$ (Right shift will be up to decimal equivalent of $\left(\mathrm{D}_{0} \mathrm{XOR} \mathrm{K}\right)$ ).

\subsection{Illustration of KVRN}

Let sender sends initial data $\mathrm{D}_{0}(00000000)$ in encrypted form using key $\mathrm{K}_{0}(00000110$ ) and one numeric value $\mathrm{m}$ (3). By the technique of KVRN as in eqn. 6 , the next keys will be generated by adding 1 (00000001), 2 (00000010) and 3 (00000011) subsequently with $\mathrm{K}_{0}=00011000$. So the next keys will be $\mathrm{K}_{0}+\mathrm{m}_{\mathrm{i}}(\mathrm{i}=1,2$ \& 3$)$. When the value of $\mathrm{m}$ will reach 3 , new key $\left(\mathrm{K}_{\mathrm{m}}\right)$ and another numeric value (n) will be exchange between sender and receiver.

\section{ANALYSIS AND COMPARISON}

For analysis and comparison of AVK, CSAVK, ASAVK, DSAVK, PROTOCOL-I, PROTOCOL-II AND PROTOCOLIII we assume two parameter explained as below:

\subsection{Analysis by randomness}

Randomness as a measure of amount of variation made between keys. The randomness for the purpose is defined as the number of bit location in which any two successive key vary. For example if:

\section{$\mathrm{K}_{\mathrm{i}}=10101010, \mathrm{~K}_{\mathrm{i}+1}=10001111$}

The randomness between two successive key is 3 . We call $\mathrm{K}_{\mathrm{i}+1}$ is random to $\mathrm{K}_{\mathrm{i}}$ by 3 .

We measure the randomness for one initial key $\mathrm{K}_{0}=$ 10101010 to perform key generation of AVKs, CSAVKs, ASAVKs, DSAVKs and for KVRN, PROTOCOL-I, PROTOCOL-II, PROTOCOL-III technique the set of initial keys are $\{10101010,01101110,11010010,10111110$, $01101111,10011011\}$, for set of initial data $\{10000101$, 1011001, , 11101, 11101011, 1100001, 1000010, 1001110, 11111, 11100010, 1100001, 1100001, 10011101, 1011001, 10011010, 10000011, 10111011, 100000, 11011110, 10111, 10001100, 1111100, 1101, 10000101, 11110001, 1011, 11101100, 11111001, 11101010, 111111, 1010110, 10011101, 10111101, 11110011, 11010011, 10110110, 1011001, 10010111, 100100, 100001, 10100, 10010000, 1011100, 10111011, 11111001, 1010001, 101111, 10100001, 11011010, 101100, 10101110, 1101110, 101011,
10011001, 1011000, 11010101, 1000010, 10110000, 101001, 1101010, 10011010, 11111100, 11001100, 11011100, 11101111, 10101111, 101011, 1000110, $11111100, \quad 11010111, \quad 1101, \quad 11010101,11010101$, 10010010, 10010000, 1111100, 10001111, 110101, 10110100, 11100100, 1011000, 10110111, 10000001, 11010011, 1101001, 1011011, ,11000000, 10001, 1001001, $10100010, \quad 10111100,11111000,100111,11100110$, 11111001, 10000001, 11111011, 1110110, 11000011, $1010010,10100110\}$

The randomness as defined was calculated by run of a programme and results so obtained are portrayed in fig.1, fig.2, fig.3, fig.4, fig.5, fig.6, fig.7 and fig.8.

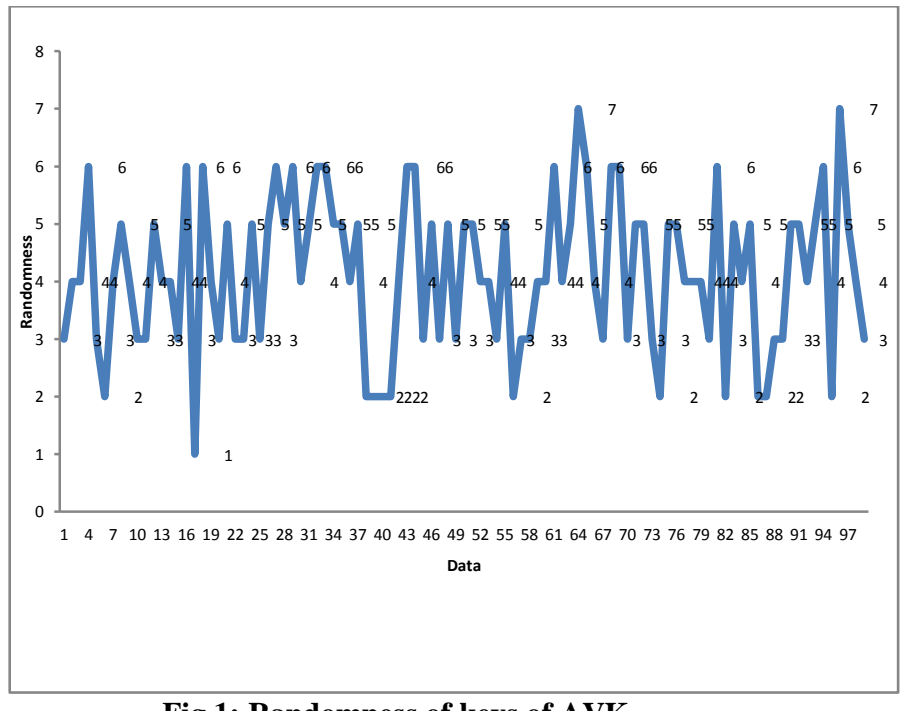

Fig.1: Randomness of keys of AVK

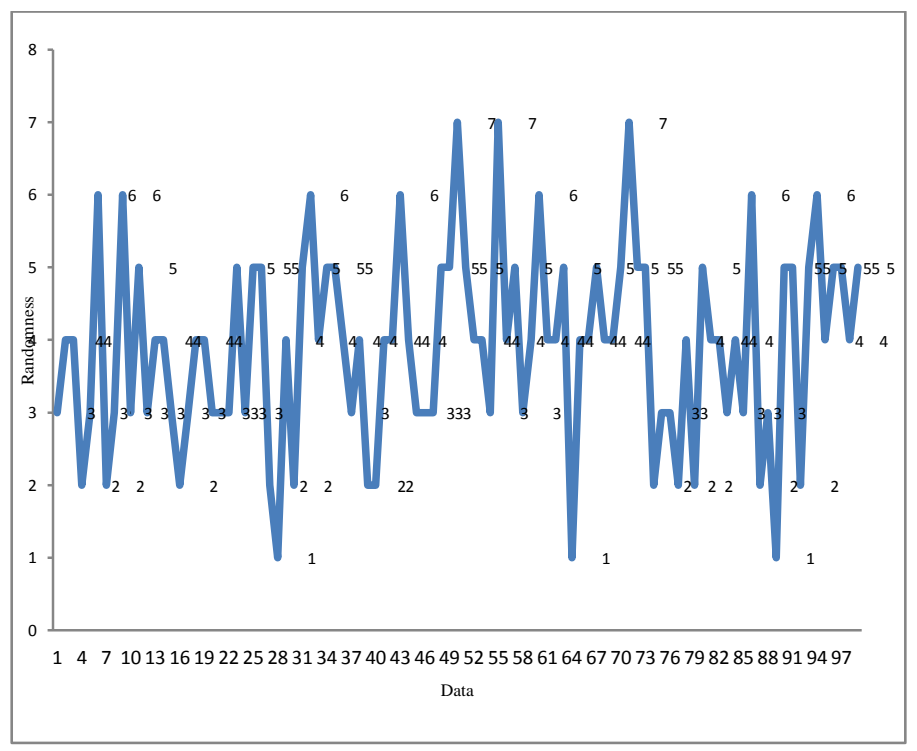

Fig.2: Randomness of keys of CSAVK 


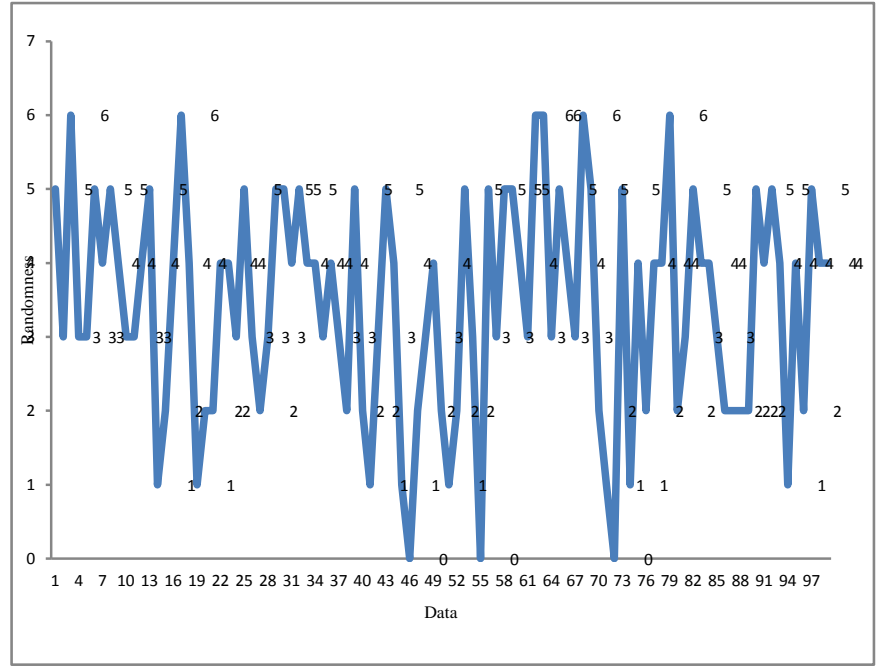

Fig.3: Randomness of keys of DSAVK

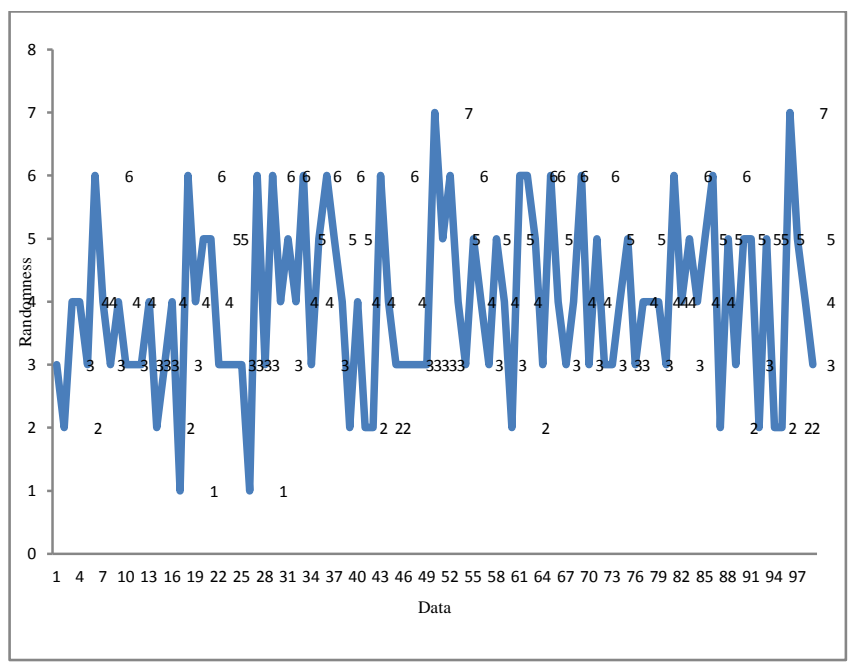

Fig.4: Randomness of keys of ASAVK

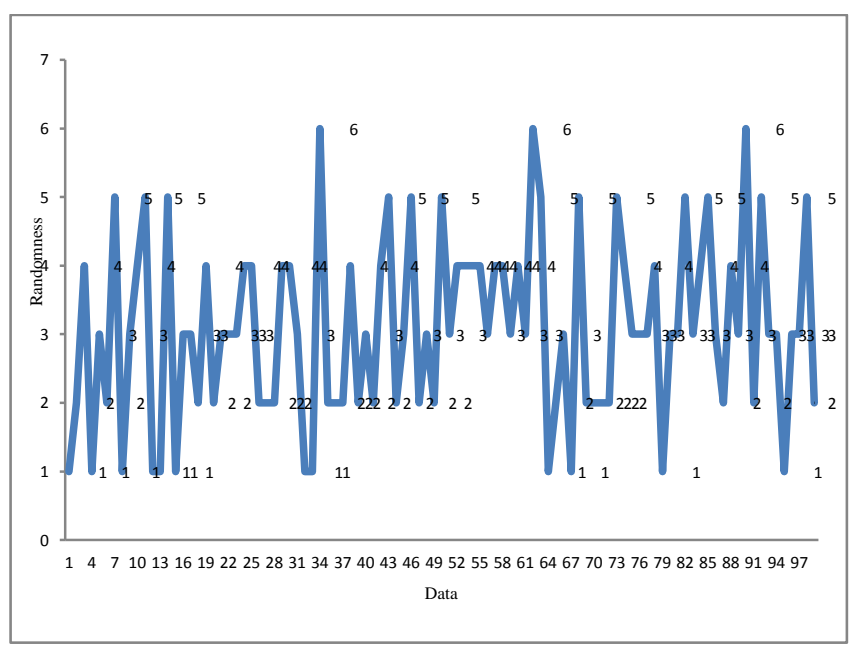

Fig.5: Randomness of keys of KVRN

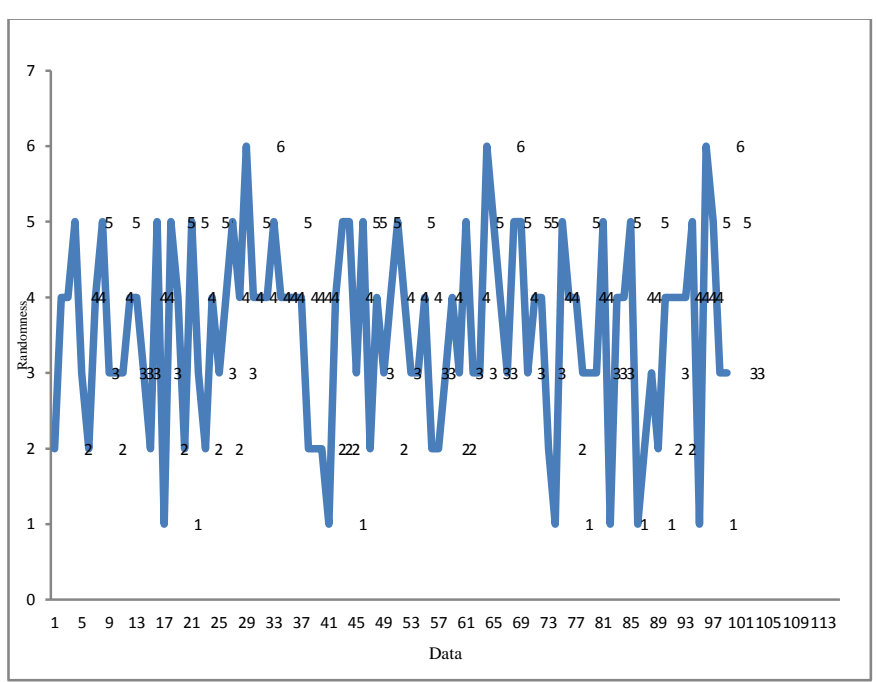

Fig.6: Randomness of keys of Protocol-I

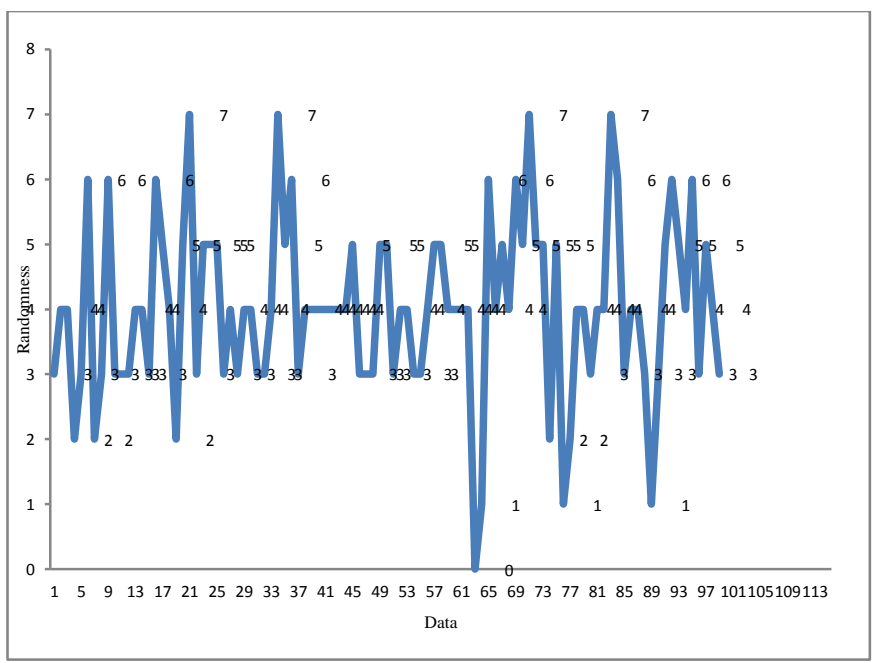

Fig.7: Randomness of keys of Protocol-II

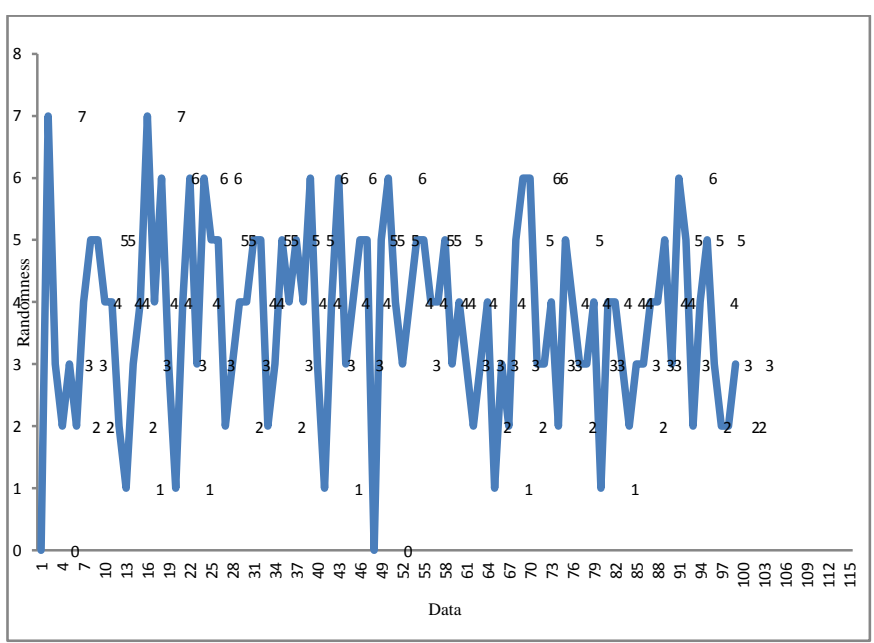

Fig.8: Randomness of keys of Protocol-III 


\subsection{Analysis by RMS}

In all previous studies the variation types of AVKs were compared in terms of absolute measure of randomness that measures the number of position in which bit differs in two successive keys. Such a measure does not reflect a consolidated parameter for comparing the techniques.

We introduce a parameter of RMS of randomness for comparison of the techniques. The measured RMS variation is shown in fig.9.

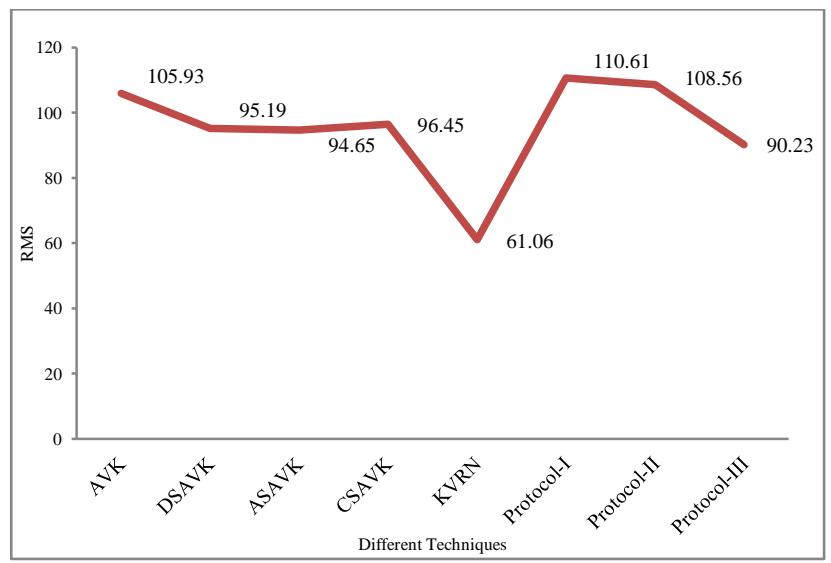

Fig.9: comparison of RMS of AVK, CSAVK, ASAVK, DSAVK, PROTOCOL-I, PROTOCOL-II and ROTOCOL-III

\section{CONCLUSIONS}

Based on result reported so far we find that so far RMS variation is concerned the proposed Protocol - I and Protocol- II are superior to other techniques. The new approach of initial key fixation by majority logic provides a confidence of application of AVK

\section{REFERENCES}

[1]. C E Shannon, "Mathematical theory of communication", The Bell System Tech J, Vol. 27,1984, pp. 379-423, 623-656.

[2]. C E Shannon, "Communication Theory of Secrecy System", The Bell System Tech J, 1949.

[3]. C.T.Bhunia, G.Mondal, and S.Samaddar, "Theory and application of time variant key in RSA and that with selective encryption in AES", 2006, Indian Engineering Congress, Kolkata.

[4]. C. T. Bhunia, "New approaches for selective AES towards tackling error propagation effect of AES," Asian Journal of Information Technology, vol.5990, pp.1017-1022,2006.
[5]. P. Chakrabarti, B. Bhuyan, A. Chowdhuri and C.T.Bhunia, "A novel approach towards realizing optimum data transfer and automatic variable key (AVK)," International Journal of Computer Science and Network Security, vol.8,no.5,May2008.

[6]. C Konar, C T Bhunia, "A novel approach towards realizing optimum Data Transfer and AVK in cryptography",International Journal of Computer Science and Network Security, Vol 8, No 5, 2008, pp. 241-250.

[7]. C T Bhunia, "New Approaches for Selective AES towards Trackling Error Propagation Effect of AES", Asian Journal of Information Technology, Pakistan, Volume 5, No. 9, pp 1017- 1022, 2006.

[8]. C T Bhunia et al, "Implementation of Automatic Variable Key with Choas Theory and Studied Thereof", J IUP Computer Science, Vol V, No 4, 2011, pp 22-32.

[9]. C T Bhunia et al, "Theories and Application of Time Variant Key in RSA and that with selective encryption in AES", Proc. EAIT, Elsevier Publications, Calcutta CSI 2006, pp 219-221.

[10]..Chakrabarty, C T Bhunia et al, "A novel approach towards realizing optimum Data Transfer and AVK in cryptography", International Journal of Computer Science and Network Security, Korea, Vol 8, No 5, May 2008, pp. 241-250.

[11].C. T. Bhunia, Swarnendu Kumar Chakraborty, Rajat Subhra Goswami, "A New Technique (CSAVK) of Automatic Variable Key in Achieving Perfect Security", 100th Indian Science Congress Association 3rd - 7th, January, 2013

[12].Rajat Subhra Goswami, Swarnendu Kumar Chakraborty, Abhinandan Bhunia, C. T. Bhunia, "New approach towards generation of Automatic Variable Key to achieve Perfect Security", 10th International Conference on Internet Technology, Next Generation, ITNG, 2013, 14th - 17th April' 2013, IEEE computer Society, CPS, pp:489-491.

[13].Rajat Subhra Goswami, Swarnendu Kumar Chakraborty, Abhinandan Bhunia, C. T. Bhunia, "Various New Methods of Implementing AVK", $2^{\text {nd }}$ International Conference on Advances in computer Science \& Engineering, CSE2013, $1^{\text {st }}-2^{\text {nd }}$ July' 2013 , Atlantis Press, pp: 149-152.

[14].Rajat Subhra Goswami, Swarnendu Kumar Chakraborty, Abhinandan Bhunia, C. T. Bhunia, "Generation of Automatic Variable Key under various approaches in Cryptography System", Communicated to Journal of the Institution of Engineers (India). 